\title{
Skyrmion Lattices Observed by High-Voltage Holography Electron Microscopes
}

\author{
Hyun Soon Park
}

Department of Materials Science and Engineering, Dong-A University, Busan, Republic of Korea.

Electron holography and Lorentz microscopy, using coherent electron waves emitted from cold fieldemission sources, provides opportunities for directly detecting and visualizing, in real space, the phase shifts of the electron waves due to the electromagnetic fields [1]. In 1992, vortices in a $\mathrm{Nb}$ superconducting thin film were observed by using a "coherent electron wave” Lorentz microscopy, simultaneously revealing their static lattice patterns and their dynamics in real time [2]. In 2009, neutron-scattering studies have shown the existence of skyrmion crystals in bulk helimagnet MnSi, by which 6-fold intensity patterns with a short lattice parameter $(\sim 17.5 \mathrm{~nm})$ were detected [3]. Two of the most important areas of study in research on the skyrmion lattice are its topological magnetic configuration and nucleation/annihilation process during magnetic phase transition. Recent Lorentz microscopy studies (2010) have revealed the magnetic configuration of skyrimon lattices and the magnetic phase diagrams for $\mathrm{Fe}_{0.5} \mathrm{Co}_{0.5}$ Si thin samples in which the skyrmion lattice constant is $90 \mathrm{~nm}$ [4]. In 2012, the skyrmion lattices with a short lattice parameter (18 $\mathrm{nm})$ in MnSi thin samples were observed by in situ observation using Lorentz microscopy [5].

Real-space imaging of skyrmion lattices has been performed by using the Fresnel (out-of-focus) method of Lorentz microscopy. Figure 1 shows the changes in the magnetic structure as a function of the applied magnetic field at $10 \mathrm{~K}$ in $\mathrm{MnSi}$ thin sample. Stripe magnetic domains, which were imaged as bright and/or dark lines, were clearly visible at zero applied magnetic field (a). Change in stripe domain contrast with increase in applied field was shown in b and c. Magnetic structures showing 6-fold symmetry appeared for magnetic field of $0.18 \mathrm{~T}$ (d) and gradual disappearance of skyrmion lattice with further increase in magnetic field was observed. Even though we reported the first direct observation of a short-period skyrmion lattice (18 nm) in a MnSi system [5], quantitative analysis of the magnetic flux flow inside and outside a skyrmion was quite difficult because of both the resolution limit (due to the defocused condition) and the unwanted artefacts (surface roughness or contamination) of thin samples.

Precise phase measurement of weak phase objects such as skyrmions is very challenging because procedures are needed for averaging the phase images and separating the electric and magnetic vector potentials. Nevertheless, the advantage of electron holography compared to Lorentz microscopy, under just-focused condition, makes it possible to visualize a quantized magnetic flux with nanometer resolution, in addition to determining its density in the vicinity of skyrmions. Here we investigated the 2D magnetic flux distributions (Fig. 3a) of skyrmion lattices in helimagnet $\mathrm{Fe}_{0.5} \mathrm{Co}_{0.5} \mathrm{Si}$ thin samples with a stepped thickness as shown in Fig. 2 and estimated the 3D structures (Fig. 3b) of the skyrmion phases by using $300 \mathrm{kV}$ and 1MV field-emission electron microscopes [6]. Our study demonstrates the potential of using a high-voltage holography electron microscope in various applications, such as the 3D visualization of magnetic fields in emergent matter systems and spintronics.

The author thanks the late Dr. A. Tonomura for his valuable discussions and all the members of the FIRST Tonomura project. This research was supported by a grant from the JSPS through the 'Funding Program for World-Leading Innovative R\&D on Science and Technology', under the programs 'Development and Application of an Atomic-resolution Holography Electron Microscope’ and 'Quantum Science on Strong Correlation’.

\section{References:}


[1] A. Tonomura, Rev. Mod. Phys. 59, 639 (1987).

[3] S. Muhlbauer et al., Science 323, 915 (2009).

[5] A. Tonomura et al., Nano Lett. 12, 1673 (2012). (2014).

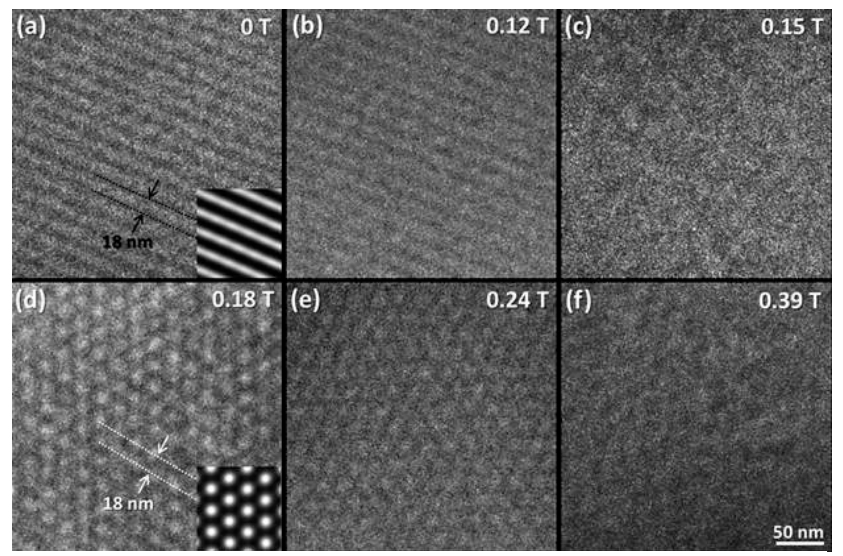

Fig. 1. Magnetic-field dependence of magnetic structure in MnSi sample (Reprinted from Ref. 5)
[2] K. Harada et al., Nature 360, 51 (1992).

[4] X. Yu et al., Nature 465, 901 (2010)

[6] H. S. Park et al., Nature Nanotech. 9, 337

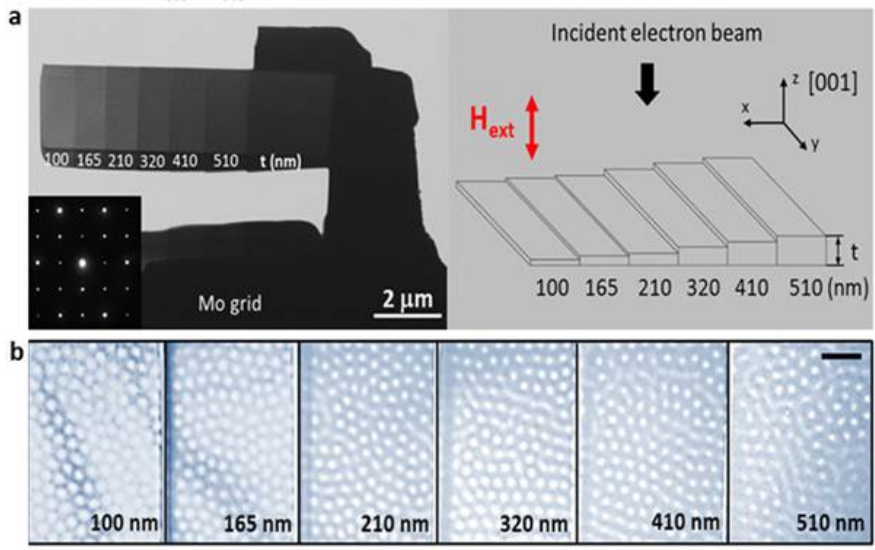

Fig. 2. Lorentz micrographs of $\mathrm{Fe}_{0.5} \mathrm{Co}_{0.5} \mathrm{Si}$ sample with a stepped thickness (Reprinted from Ref. 6)
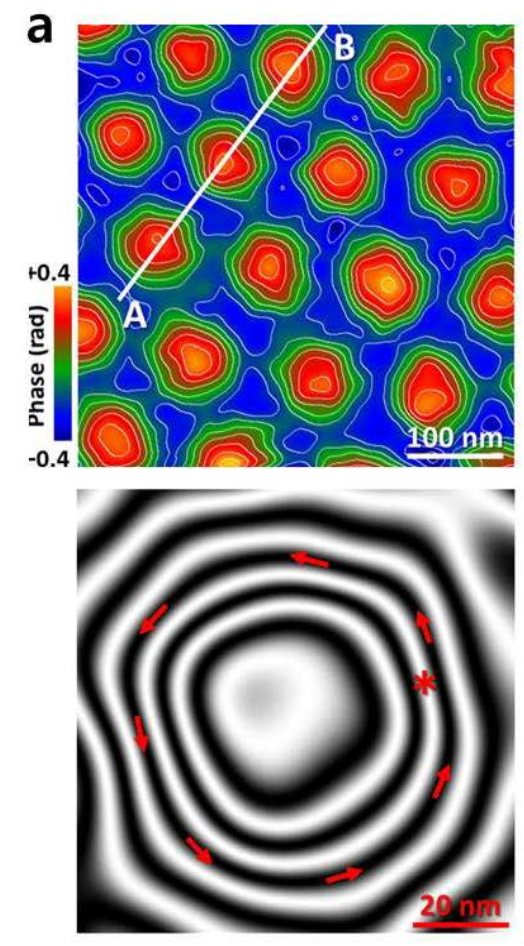
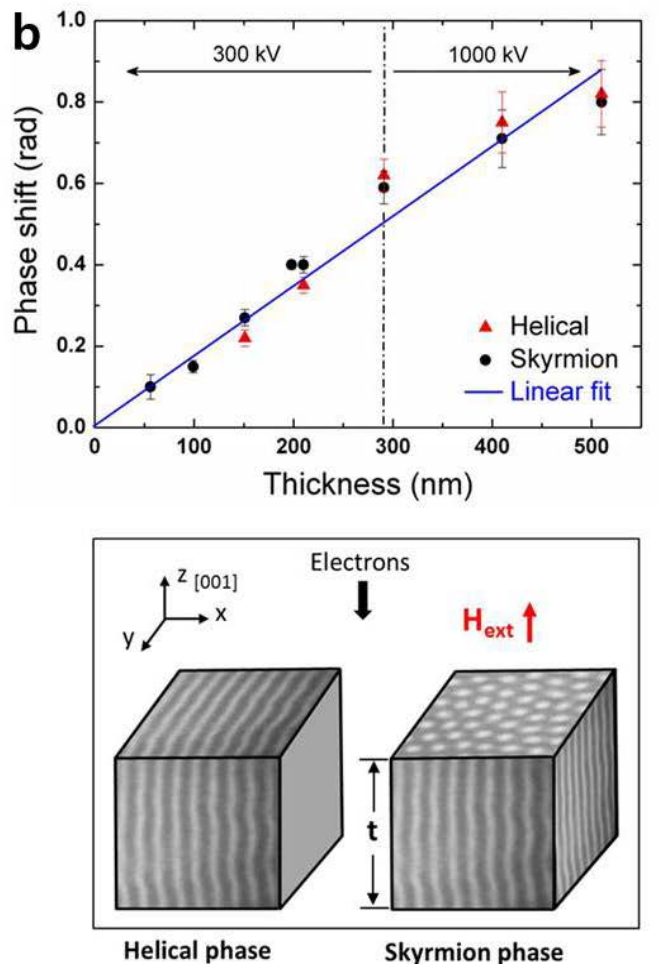

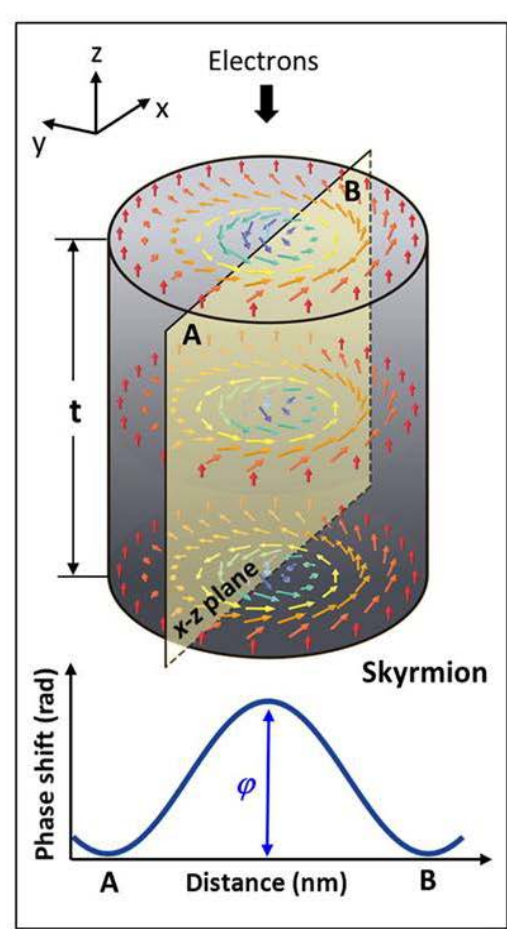

Fig. 3. 2D phase maps and 3D structure of skyrmion lattices in $\mathrm{Fe}_{0.5} \mathrm{Co}_{0.5} \mathrm{Si}$ thin sample. (a) Magnetic flux maps showing the phase shift due to magnetic vector potential. The difference between equi-phase lines corresponds to 0.1 rad. (b) Electron phase shifts of the helix and skyrmion as a function of sample thickness (carried out using 300 and 1,000 kV microscopes). Schematic illustration showing the 3D spin structure of a skyrmion and its phase shift profile for line A-B (in the $\mathrm{x}-\mathrm{z}$ plane), integrated through specimen thickness $t$ (Reprinted from Ref. 6). 